\title{
Finanzielle Anreize zur Complianceförderung
}

\author{
Monetary Incentives to Improve Compliance
}

Bibliografie

DOI 10.1055/s-0029-1220399

Psychiat Prax 2009; 36:

258-260

(c) Georg Thieme Verlag KG

Stuttgart · New York .

ISSN 0303-4259

Korrespondenzadressen

Dr. Werner Kissling

Klinik und Poliklinik für

Psychiatrie und Psychotherapie

der TU München

Ismaninger Str. 22

81675 München

w.kissling@|rz.tum.de

\section{Dr. Michaela Stiegler}

Klinik und Poliklinik für

Psychiatrie und Psychotherapie

der TU München, Klinikum

rechts der Isar

Ismaninger Str. 22

81675 München

m.stiegler@|rz.tum.de

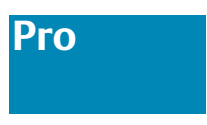

Als „Compliance (Folgsamkeit, Therapietreue)“" wird die Bereitschaft und Fähigkeit eines Patienten bezeichnet, den medizinischen Verordnungen zu folgen. Der modernere Begriff der „Adherence (Einhaltung)“ beinhaltet zusätzlich, dass der Patient bei der Auswahl der Therapie mit einbezogen wurde und ihr zugestimmt hat. Im Kontext der vorliegenden Fragestellung kann es natürlich nur um „Adherence“ gehen, also darum, ob man Patienten durch finanzielle Anreize dazu motivieren kann und soll, eine in Abstimmung mit ihnen vereinbarte Therapie so lange wie vereinbart auch tatsächlich durchzuführen.

\section{Warum brauchen wir finanzielle Anreize?}

Non-Adherence bei der Langzeitbehandlung chronischer Erkrankungen ist derzeit in den entwickelten Ländern das gravierendste Gesundheitsproblem [1]: Die Hälfte aller chronisch erkrankten Patienten nehmen ihre Medikamente nicht ein. Die Folgen sind hohe Rückfall- und Wiederaufnahmeraten, eine Verschlechterung der Gesamtprognose und massives persönliches Leid bei den betroffenen Patienten und ihren Familien. Ein WHO-Report [1] zitiert Studien, nach denen in den USA allein im Bereich der HerzKreislauf-Erkrankungen jedes Jahr ca. 125000 Menschen an den Folgen ihrer Non-Adherence sterben. Selbst nach Organtransplantationen nehmen 20\% der Patienten ihre Immunsuppressiva nicht ein und sterben deshalb oder benötigen ein zweites Organ [2]. Bei psychiatrischen Patienten ist die Noncompliance noch deutlich höher als bei somatischen Erkrankungen: Sie ist die Ursache für die Hälfte aller stationären Wiederaufnahmen bei schizophrenen und depressiven Patienten [3] und hauptverantwortlich für die hohen Behandlungskosten bei diesen Erkrankungen. Insgesamt kostet die Noncompliance in Deutschland jährlich ca. 10 Milliarden Euro [3] und dieses Geld fehlt dann für dringend benötigte medizinische Leistungen an anderer Stelle.

Aus allen diesen Untersuchungen geht klar hervor, dass Noncompliance eines der wichtigsten Gesundheitsprobleme ist und dass dieses Problem aus medizinischen, ethischen und finanziellen Gründen dringend und rasch gelöst werden muss. Mit allen bisher versuchten Strategien (Fortbildungsmaßnahmen, Qualitätsmanagement, Psychoedukation etc.) ist diese Lösung aber bis jetzt nicht gelungen.

Wenn es über längere Zeit nicht gelingt, ein drängendes Gesundheitsproblem mit den herkömmlichen Mitteln zu lösen, ist es gerechtfertigt, nach neuen, unorthodoxen Lösungswegen Ausschau zu halten. Wenn man - wie im vorliegenden Fall - dann glaubt, eine Lösungsmöglichkeit gefunden zu haben, muss man ihren potenziellen Nutzen gegen mögliche Risiken abwägen, evtl. noch die Praktikabilität und Finanzierbarkeit ausloten und kann dann die neue Lösung in einer Pilotstudie ausprobieren. Dieser Abwägungsprozess kann verkürzt werden, wenn die ins Auge gefasste Lösung bereits in anderen Ländern oder bei anderen Indikationen erfolgreich erprobt wurde. Aus unserer Sicht sind alle diese Voraussetzungen im vorliegenden Fall erfüllt.

Finanzielle Anreize sind wirksam: In zahlreichen Studien konnte gezeigt werden, dass durch finanzielle Anreize ein gesundheitsförderliches Verhalten und eine bessere Adherence bewirkt werden können [4-6], vor allem bei somatischen Erkrankungen (Tuberkulose, HIV), aber auch bei Suchterkrankungen und anderen psychiatrischen Krankheiten [4].

Finanzielle Anreize haben keine unzumutbaren Risiken: Wie jede Intervention birgt auch das Anbieten finanzieller Anreize gewisse Risiken (Abbau intrinsischer Motivation, Störung der ArztPatienten-Beziehung, Verführung zu ungewolltem Verhalten, Missbrauchspotenzial, Geldver- 
schwendung), diese sind aber selten und erscheinen angesichts der Chancen (Vermeidung von Rückfällen und evtl. sogar Todesfällen, Einsparung von Ressourcen, die dringend für andere Patienten benötigt werden etc.) vertretbar.

Finanzielle Anreize sind praktikabel und finanzierbar: Mehrere Arbeitsgruppen haben finanzielle Anreize im Rahmen von Complianceprogrammen erprobt und fanden, dass sie praktikabel, kosteneffektiv und wirksam sind $[4-6,8]$.

\section{Resümee}

Wir halten es beim derzeitigen Wissensstand für gerechtfertigt, finanzielle Anreize anzubieten, um gesundheitsförderliches Verhalten zu verstärken. Die zu diesem Thema bisher vorliegenden Studien und praktischen Erfahrungen lassen erwarten, dass solche Anreize positive Wirkungen erzielen und keine unvertretbaren Risiken aufweisen. Das gilt auch für psychiatrische Patienten [4]. Wenn der Patient bei der Wahl der Therapie mitbestimmen durfte, dann sind aus unserer Sicht finanzielle Anreize ethisch vertretbar, weil sie nicht dazu dienen, den Patienten zu einer eigentlich nicht gewünschten Therapie zu verleiten, sondern ihn nur dabei unterstützen, seine Therapieziele $\mathrm{zu}$ erreichen und die Behandlung so lange wie vereinbart durchzuhalten. Durch kurzfristige finanzielle Anreize wird es dem Patienten erleichtert, seine langfristigen Therapieziele (Rezidivfreiheit, Abstinenz etc.) zu erreichen.

Wie immer bei der Einführung einer neuen Intervention sind natürlich viele Fragen noch nicht abschließend geklärt. Angesichts der Dringlichkeit des Adherence Problems und des Fehlens gravierender Risiken sollte uns das aber nicht davon abhalten, diese neue Strategie in Pilotstudien bei geeigneten psychiatrischen Indikationen weiter zu erproben. Vor einem routinemäßigen Einsatz von finanziellen Anreizen sollten allerdings die wichtigsten offenen Fragen noch umfassender untersucht werden. Aus unserer Sicht sind dies die Folgenden:

Wirksamkeit, Risiken und Praktikabilität von Bonussystemen bei psychiatrischen Störungen, insbesondere bei Suchterkrankungen und bei der rezidivprophylaktischen Langzeitbehandlung von Schizophrenie und Depression? Hierzu müssen rasch noch mehr qualitative und quantitative kontrollierte Studien durchgeführt werden. Insbesondere sollten dabei noch folgende Fragen genauer beantwortet werden:

- Optimale „Dosis“, Zeitpunkt und Dauer finanzieller Anreize

- Wirksamkeit und Kosteneffektivität in naturalistischen kontrollierten Studien

- Bei welchen Patienten bzw. Indikationen sind finanzielle Anreize indiziert, bei welchen nicht?

- Welche Nachteile haben finanzielle Anreize?

Wir haben uns nach sorgfältiger Abwägung der Chancen und Risiken (vgl. [4] und Stiegler in diesem Heft) vor einem Jahr entschlossen, finanzielle Anreize (Fahrtkostenerstattung und Einkaufsgutscheine) im Rahmen unserer Compliance Programme für ambulante Patienten mit Schizophrenie oder Depression probeweise anzubieten. Im Sinne eines vorsichtigen Vorgehens haben wir in einer ersten Stufe allerdings nur die Teilnahme an complianceverbessernden Maßnahmen (z.B. Psychoedukation) belohnt und noch nicht direkt die Adherence mit einer bestimmten Therapie. Da wir bis jetzt keinerlei Nachteile oder Risiken feststellen konnten und wir im Prä-Post-Vergleich den Eindruck gewannen, dass finanzielle Anreize die Teilnahmerate an complianceverbessernden Maßnahmen erhöhen, sollen jetzt in einer prospektiven Kontrollgruppenstudie einige der oben genannten Fragen noch genauer untersucht werden. Wenn sich dabei Prak- tikabilität und Wirksamkeit von finanziellen Anreizen weiter bestätigen, könnte in einem zweiten Schritt auch direkt die Wirksamkeit finanzieller Anreize auf die Adherence gegenüber einer bestimmten Therapie untersucht werden.

\section{Kontra}

Ohne jede Frage ist mangelhafte medikamentöse Compliance seitens unserer psychiatrischen Patienten eine der Hauptursachen für unbefriedigende Therapieerfolge: So halten sich ungefähr zwei Drittel der Patienten mit Schizophrenie zumindest teilweise nicht an das verordnete Medikationsregime - trotz Entwicklung der besser verträglichen Second-Generation-Antipsychotika - was bekanntlich zu hohen Wiederaufnahmeraten führt $[9,10]$.

Eine durchschlagende Verbesserung der Compliance konnte bisher trotz unterschiedlichster Ansätze noch nicht erreicht werden und stellt damit eine der größten Herausforderungen an unser Gesundheitswesen dar. Eine Option wäre „Tablette gegen Bares“ - doch geht diese Gleichung wirklich auf?

Aussagekräftige wissenschaftliche Daten über eine mögliche positive Wirkung von Geldzahlungen auf die medikamentöse Compliance speziell von psychiatrischen Patienten gibt es bisher nicht. Auch wenn die raren Studien mit Patienten aus anderen medizinischen Gebieten positive Effekte dieser Intervention auf die Compliance zeigen konnten, ist fraglich, ob diese Ergebnisse so einfach auf den Bereich Psychiatrie übertragen werden können [11]. Allein die aktuelle Datenlage macht es somit schwierig, von Erfolgen durch finanzielle Boni bei Patienten mit psychischen Erkrankungen auszugehen.

\section{Offene Fragen}

Völlig ungeklärt ist die Finanzierung eines solchen Bonussystems. Denn nicht nur Patienten mit psychischen Erkrankungen hätten dann das Recht, in den Genuss von Geldzahlungen zu kommen, sondern auch die Vielzahl von Menschen mit anderen chronischen Leiden wie Diabetes oder Hypertonie, die über Jahre oder Jahrzehnte auf die Behandlung mit Medikamenten angewiesen ist. Über die erforderliche Höhe der Zahlungen lassen sich ebenfalls keine wissenschaftlich überprüften Aussagen treffen - reichen 10 Euro pro Monat aus oder muss das 10-Fache geboten werden, um die Compliance zu beeinflussen? Was für den einen eine Verdoppelung seiner Einnahmen bedeuten kann, ist für den anderen der übliche Stundenlohn. Grundsätzlich müsste aber jeder Patient für die gleiche Leistung die gleiche Vergütung erhalten, einen adäquaten Betrag zu finden wäre also schwierig. Die notwendige Dauer der Geldzuwendungen ist ebenfalls unklar. Ergebnisse aus bereits durchgeführten Studien deuten darauf hin, dass der positive Effekt nach Beendigung der Zahlungen verschwindet und es hinsichtlich der Compliance zu einem Rebound-Effekt kommt [12]. Um auf Dauer eine Wirkung zu erreichen, müssten chronisch Kranke also über Jahrzehnte finanziell motiviert werden. Doch die Töpfe des öffentlichen Gesundheitswesens sind leer, und es ist völlig unklar, wer die erwartungsgemäß hohen Aufwendungen bestreiten soll.

Insgesamt wäre die Organisation einer gerechten Verteilung der Zahlungen fast unmöglich. Wer entscheidet, welche Behandlung respektive welche Medikation für eine bestimmte Diagnose indiziert ist und damit auch vergütet wird? Evidenzbasierte Behandlungsleitlinien, die die Grundlage für bezahlte Tabletteneinnahme bilden könnten, existieren nur für vereinzelte Diagnosen, und eine Anwendung dieser Empfehlungen ist in Deutschland nicht verbindlich [13]. So liegt es im Ermessen des behandelnden Arztes, Kombinationen aus unterschiedlichen Medika- 
menten für eine bestimmte Erkrankung zu verordnen. Wird der Patient dann für die Einnahme jedes einzelnen Medikaments bezahlt oder für das Befolgen des kompletten Therapieregimes? Spinnt man diesen Gedanken weiter, könnte eine finanziell lohnende Polypharmakotherapie möglicherweise sogar dazu führen, dass manche Patienten die Verordnung von immer mehr Medikamenten einfordern, um höhere Zahlungen zu erhalten. Oder eine Besserung der Symptomatik wird dem Behandler verheimlicht, was im Bereich Psychiatrie nicht unmöglich ist, um ein Absetzen der Medikation hinauszuzögern.

Eine Kontrolle der tatsächlichen Compliance wäre ohnehin schwierig und die Organisation äußerst aufwendig. Sich nur auf die Angaben der Patienten zu verlassen, genügt bekanntlich nicht. Methoden zur Messung der korrekten Tabletteneinnahme wie etwa das Medication Event Monitoring System (MEMS), bei dem die Häufigkeit des Öffnens des Tablettenspenders elektronisch gemessen wird, oder der Pill Count, bei dem lediglich die eingenommenen Dosen abgezählt werden, sind nur bedingt aussagekräftig. Beweisend für tatsächlich eingenommene Medikation wären allein Blutuntersuchungen, doch auch hier kann manipuliert werden, vom zusätzlichen Zeitaufwand für uns Ärzte und der unangenehmen Rolle als Kontrolleur der Patienten ganz zu schweigen. Die therapeutische Allianz würde unter diesen Kontrollen sicher leiden.

\section{Patientenperspektive}

Wie stellen sich Geldzahlungen für Compliance eigentlich aus Sicht der Patienten dar? „Mein Arzt bietet mir Geld, damit ich etwas angeblich Positives für meine Gesundheit tue, Medikamente einnehme, von denen eigentlich behauptet wird, dass sie mir helfen. Warum sollte er das tun, wenn die positiven Seiten tatsächlich überwiegen würden? “ Der Verdacht könnte für den Patienten naheliegen, eine eigentlich negative Maßnahme solle ihm durch finanzielle Entschädigung schmackhaft gemacht werden. Tatsächlich sind eventuelle Spätschäden durch neuere Medikamente noch nicht absehbar - was, wenn das gebotene Geld den Patienten letztlich dazu gebracht hat, sich auf lange Sicht zu schaden?

Eine ideale Therapie besteht aus einer vertrauensvollen Arzt-Patienten-Beziehung, in der der aufgeklärte Patient im besten Fall zusammen mit seinem behandelnden Arzt unterschiedliche Therapieoptionen abwägt und dann gemeinsam entschieden wird, welche Behandlung momentan angebracht ist. Ziel neuerer Methoden wie dem Shared-Decision-Making ist es, diesen Ansatz in die Routinebehandlung zu integrieren [7].
Der Patient soll also möglichst frei entscheiden können und als gleichwertiger Partner gewertschätzt werden. Ziel müsste demnach sein, die Selbstwirksamkeit der Betroffenen zu erhöhen. Durch Bieten von Geld für ein bestimmtes, erwünschtes Verhalten wird der Patient jedoch auf gewisse Weise manipuliert, der Arzt versucht, sich das erwünschte Verhalten zu „erkaufen“. Die Entscheidungsfähigkeit des Patienten kann dadurch massiv beeinflusst werden, denn das manipulative Potenzial des Geldes darf weder im Alltag noch im besonders sensiblen Bereich einer medizinischen Behandlung unterschätzt werden.

Viele Fragen bleiben offen, einige Bedenken bestehen, sowohl zur Wirksamkeit von Geldzahlungen auf die Compliance und zur Finanzierung als auch organisatorischer und nicht zuletzt ethischer Natur, sodass vor einer Implementierung dieser Maßnahme die Vor- und Nachteile gewissenhaft abzuwägen sind.

\section{Literatur}

1 World Health Organisation. Adherence to long-term therapies: evidence for action. (www.connected-health.org/programs/medicationadherence.aspx) 2003

2 Rovelli M, Palmeri D, Vossler E et al. Noncompliance in renal transplant recipients: evaluation by socioeconomic groups. Transplant Proc 1989; 21: 3979-3981

3 Seemann U, Kissling W. Volkskrankheit „Noncompliance“. Psychoneuro 2008; 34 (9): 405-409

4 Stiegler $M$. Telefonisches Compliance-Monitoring und Bonusprogramme. Psychoneuro 2008; 34 (9): 417-420

5 Marteau TM, Ashcroft RE, Oliver A. Using financial incentives to achieve healthy behaviour. BMJ 2009; 338: b1415

6 Giuffrida A, Torgerson DJ. Should we pay the patient? Review of financial incentives to enhance patient compliance. BMJ 1997; 315: 703-707

7 Hamann J, Cohen R, Leucht S et al. Shared decision making and longterm outcome in schizophrenia treatment. J Clin Psychiatry 2007; 68 (7): 992-997

8 Kissling W. Das Münchner Modell. Psychoneuro 2008; 34 (9): 410-415

9 Leucht S, Barnes TR, Kissling $W$ et al. Relapse prevention in schizophrenia with new-generation antipsychotics: a systematic review and exploratory meta-analysis of randomized, controlled trials. Am J Psychiatry 2003; 160 (7): 1209-1222

10 Rummel-Kluge C, Schuster T, Peters S et al. Partial compliance with antipsychotic medication is common in patients with schizophrenia. Aust N Z J Psychiatry 2008; 42 (5): 382-388

11 Bock NN, Sales RM, Rogers T et al. A spoonful of sugar ...: improving adherence to tuberculosis treatment using financial incentives. Int J Tuberc Lung Dis 2001; 5 (1): 96-98

12 Rigsby MO, Rosen MI, Beauvais JE et al. Cue-dose training with monetary reinforcement: pilot study of an antiretroviral adherence intervention. J Gen Intern Med 2000; 15 (12): 841-847

13 Stiegler M, Rummel C, Wahlbeck $K$ et al. European psychiatric treatment guidelines: is the glass half full or half empty? Eur Psychiatry 2005; 20 (8): 554-558 